\title{
Seamless Integration of Chemical and Biological Engineering In The Undergraduate Curriculum
}

\section{Howard Saltsburg, Maria Flytzani-Stephanopoulos, David Kaplan, Gregory Botsaris, Kyongbum Lee}

\author{
Department of Chemical \& Biological Engineering \\ Tufts University \\ Medford MA
}

\section{Introduction}

Chemical engineering has evolved over the past one hundred years from a combination of chemistry and mechanical engineering into a discipline that has developed its own unique and powerful paradigm. The essential features of the modern period began when Arthur D. Little, in 1915, developed the concept of "unit operations" ". The basis of this approach was the analysis of existing chemical processes and the realization that it was possible to decompose the process into a set of "nearly independent units" with a limited number of inputs and outputs for each "unit". Subsequently, by including equilibrium and rate processes in the analysis at all scales, ranging from the molecular to the industrial plant, the paradigm of chemical engineering evolved to its present state. It provides a powerful set of tools for such unit operation analyses. With this approach, it is then possible to synthesize a process by combining these units with proper attention to mass and energy balances for the entire system as well as for the individual units. Each unit can be well characterized individually and in detail. The foundation for this approach is a reasonably clear understanding of basic chemistry, transport processes, and their interaction within a unit. During the development of the chemical engineering paradigm, the interaction of traditional chemistry and chemical engineering proved to be of benefit to both disciplines. Like all engineers, chemical engineers apply a systems approach to problem solving and produce practical and timely designs in the absence of complete information. What distinguishes them from other engineers is that they are deeply rooted in the chemical sciences.

Curricula in chemical engineering departments reflect this unit operations approach. While drawing heavily from problems of the petrochemical industry, commodity chemicals, and polymers, the core courses were adequate to prepare chemical engineers for careers in emerging areas of energy and environmental engineering, semiconductor manufacturing, and the diverse requirements of the pharmaceutical industries. Elective courses in these technologies were straight forward applications of the paradigm, as they were based on the same chemical engineering core; namely, thermodynamics, reaction kinetics, and transport phenomena. More importantly, over the years, when chemical engineering departments brought into the curriculum advanced materials, combustion and fuel engineering, biotechnology, or environmental engineering, that step did not require revision of the core and it did not necessitate complex 'tracks'. The paradigm was powerful enough to integrate those new areas, without a change in the core curriculum as the Proceedings of the 2003 American Society for Engineering Education Annual Conference \& Exposition Copyright (C) 2003 American Society for Engineering Education 


\section{Session 2613}

necessary subject matter was a derivative of the core material.

In the late '90s, momentous advances in the field of biology occurred and the prospect of large scale bio-based industrial processes resulted in calls for changes in the way we prepare scientists and engineers. The immediate conclusion is that the traditional separation of biology from the physical sciences and engineering must be eliminated. The mind set of the chemical industry itself is changing, with biology beginning to look like a major vehicle for the next 'quantum leap' of this industry as it becomes a life sciences-based one.

\section{Rationale for Chemical and Biological Engineering}

Although departments of "Agricultural and Biological Engineering" exist, their basic focus is on macroscopic biology issues in agriculture. This approach has begun to change with the introduction of genetic engineering into that discipline. "Bioengineering" was the obvious next candidate. Despite its being the center of attention in recent years, "bioengineering" is not a new field. It emerged more than 40 years ago, mostly in electrical engineering departments concerned with imaging, radiology, signal processing and device control issues. In a latent state for most of the time, bioengineering has gradually accommodated a variety of research activities in biomechanics, biomaterials, prostheses and medical devices, all activities oriented towards medical applications and with very little common intellectual content. This broadened the base of the field but at the cost of a very diffuse, weak and ill-defined curriculum. As a result, there is no such thing as a bioengineering core and educational programs invariably resort to tracks in order to serve the needs of the diverse constituents of bioengineering. Furthermore, the biomedical industry typically connected with bioengineering is rather small and centered around imaging and medical devices.

In recent years new 'Bioengineering' entities have appeared, initially as interdisciplinary programs, but with increasing frequency as 'departments' attempting to capture the loosely defined 'fusion of biology with engineering'. If one examines the curricula of these entities, it is apparent that much of the course structure is based on traditional core chemical engineering with the modifier "biological" inserted in front of the subject title ${ }^{2}$. Yet the organizational structure to fold biology into the curriculum at every level from molecular and cellular to large-scale industrial is already in place within the paradigm of the chemical engineering discipline. This is a natural progression for chemical engineering. With the inclusion of biology as a foundation, the focus of the material can be narrowed to applications of chemistry and molecular and cellular biology to problems of biotechnology, and medicine and that can be best served by taking advantage of the existing intellectual core of chemical engineering. Based on this growing interest, in the year 2000, with the full support of the University administration, the department took the bold step of renaming itself "Chemical \& Biological Engineering", the first formal recognition in the United States of the basic importance of biology as an enabling science in the chemical engineering core. The decision not to create a department of bioengineering at that time was made in part because it was difficult to identify a stand-alone fundamental core for such a department.

Thus we plan to define the required revisions necessary to fully encompass 'biological' into chemical engineering curricula so as to produce an engineer equipped to work in many fields. 


\section{Session 2613}

Employing the time-tested paradigm used so successfully before, biological engineering can be made an integral part of chemical engineering at the undergraduate level. In particular, the integration will be transparent and appear as a natural component in the courses rather than as an add-on.

\section{Proposed Approach}

As noted above, the momentous advances in biology and the clear importance of translating that knowledge into processes of industrial magnitude has led recently to a proliferation of "Bio-X" departments. These entities are critically dependent on core $\mathrm{ChE}$ material but are narrowly focused. Some ChE departments have developed complete curricula based on specific industries (e.g. UCLA and semiconductor manufacturing ${ }^{3}$ ). However, none of these groups can demonstrate a unique general core of material that distinguishes them from other engineering disciplines ${ }^{4}$. This lack of a unique core and excessive specialization is not an approach designed to educate an individual capable of working in any chemical and biologically related field nor is it appropriate for the long-term development of a department.. The approach to curriculum revision that we propose here is neither a stand-alone modular approach, nor some additive combination of biology and chemical engineering, but a seamless integration of biology into the curriculum. The net result will be that biology becomes the fourth leg of the core support structure, joining chemistry, physics, and mathematics. Approaches to issues raised by the specific problems of biological engineering are included naturally as a part of the paradigm in the same manner as chemical issues are considered. Just as the interaction of traditional chemistry and chemical engineering was mutually beneficial, the same type of benefit will accrue to biology and biological engineering united under the paradigm. The overall effect is to change the content but not the methodology of analyzing problems while maintaining the flexibility of the department to embrace new technologies.

\section{Implementation}

At the outset, students and faculty need to become familiar with the basic concepts of modern biology just as they are with basic chemistry, physics, and mathematics. An introductory biology course is now required of all Chemical and Biological Engineering students at Tufts, and we plan to enrich the chemistry component by requiring one semester of biochemistry for all students.

The heart of the proposal is the development of a learning matrix in which we broaden the base of systems to be studied, beginning with a first year course, and following these systems through the entire core curriculum. In the past, the Chemical Engineering curriculum has been dominated by analyses of systems of interest to the industry served by the discipline. These include petroleum refining, chemical and petrochemical processing, commodity chemicals, and polymers. In the scheme proposed here, we will consider in parallel two biological and three chemical systems which illustrate differences in scale and content and demonstrate the applicability of the general ChE paradigm to each of them. In such a side-by-side comparison, the issues of understanding and describing these very different systems as well as the universality of the ChE paradigm in addressing these problems can be demonstrated. The role of equilibrium, kinetics, reactors, transport, control, separation, materials, and balances common in the traditional program can be 


\section{Session 2613}

demonstrated for each of these systems. By continuing to focus on these systems throughout the core courses, we will establish a "thread" that connects all these courses and continually places the courses in context, something lacking in traditional programs. This concept of a "threaded curriculum" has been explored previously in a laboratory context ${ }^{5}$ and the extension to the full curriculum is viable. As an example, by considering a biological cell, a biological organ, a chemical plant with reaction complexity (e.g. polymer production), a modern material production (e.g. electronic grade silicon, aluminum), and a commodity chemical process (e.g. $\mathrm{NH}_{3} \mathrm{Or}_{2} \mathrm{SO}_{4}$ ) in parallel, one can demonstrate the overall applicability of the approach exemplified by the $\mathrm{ChE}$ paradigm, and with these five systems illustrate scale and complexity. This global examination places the curriculum in context for all of these systems.

Thus, using the same paradigm, we can integrate biological systems into the course work in a natural manner, remove artificial distinctions, and illustrate both the commonality and differences among these systems. Using this approach, proper attention can be paid to other important aspects of chemical engineering practice such as fuels, catalysts, and advanced materials. It is vital to develop a broad base of fundamental technical capability as the requirements for future industrial discovery and processing are unknown. This approach is not "combining" such as a stand-alone modular approach suggests but is a true seamless integration of the material based on the constant comparison. There is a need to place the material in context using the familiarity of students with the world around them both from the synthesis point of view as well as the place of such systems in their experience. This requires that in the beginning courses these selected systems be presented in the form of design questions (in the broadest sense) to place material in context.

To make this work,

- Students and faculty need to become familiar with basic biology just as they are with basic physics, chemistry, and mathematics at least at the level of cell function and biochemistry.

- Team teaching utilizing faculty experts in appropriate areas is one approach but it cannot be simply based on modules. We need to remove artificial distinctions. Although sharing each lecture is time intensive, some sense of integration must be transmitted to students.

- Laboratories are to be used to supplement course work. Students will not prove or disprove fundamental laws nor challenge well established bodies of data. They can learn new material (e.g. genetics as part of a lab) but there is a need to distinguish technique- training from new material and balance those issues.

- For the purposes of this proposal, it is important to carry out an analysis of the "units" that make up a biological system, be it a cell, an organ, or an organism to demonstrate that the same paradigm that works so well for traditional $\mathrm{ChE}$ will be applicable to these more complex entities. Given the uncertainty associated with this approach, it will be valuable for students to see what problems exist when this decomposition analysis is applied to cells and organs as compared to applications to more traditional ChE systems. It is important to see what can and cannot be done at this time. The simple "single cell" models with complex chemistry and simple input/output streams developed in the past are inadequate given the current knowledge, but it is important to know why ${ }^{6}$

Proceedings of the 2003 American Society for Engineering Education Annual Conference \& Exposition Copyright (C) 2003 American Society for Engineering Education 


\section{Session 2613}

- Finally, it is important to maintain the quantitative aspects of the program as the biological systems are explored. We do not envision embracing the biotechnology approach described by Rehm and Preve ${ }^{7}$ as "the use of biological processes within the framework of technical operations and industrial production. It is an application -oriented science of microbiology and biochemistry ...with engineering overtones". The program should not train process operators but should educate independent thinkers who are able to deal with new problems as well as the old ones. Thus, there is a core and based on that core are the derivatives: bio processing, chip manufacture, commodity chemical production, petroleum processing, and polymer production as examples.

\section{Details: curriculum structure}

We propose to modify each year of the curriculum sequentially. The course restructure will begin in the second year and in each successive year the new curriculum will be introduced. You cannot expect seniors to suddenly have the required background so the stage wise introduction is appropriate. AIChE has recognized the need for flexibility in required chemistry courses; this allows us to use a biochemistry course to satisfy one of the three advanced chemistry requirements. The biology course, already in our curriculum, satisfies the natural science elective. As a result, the new curriculum will satisfy ABET and leave unchanged the approval of the program at Tufts that was received in the year 2000. This restructuring actually moves the program further toward the 2000 guidelines. The content of concentration courses will be changed to reflect the integration. In the future, we expect ABET to establish criteria for a degree in Chemical and Biological engineering. We will be in touch with them regarding our experience to aid in establishing the appropriate criteria.

First Year

The typical first year program consists of the equivalent of 9 full credit courses but of particular importance to this proposal is the use of half-credit engineering electives for first year students. Tufts offers a wide range of topics, all of which are designed to present engineering in a context familiar to first year students. In ChBE, topics have included "Unmixing Mixtures" using clothes washing as the context, "Your Car" examining the materials of automobile construction, and "Microbrewery Engineering" examining bioprocessing. We propose to provide another course in this vein offering the seamless integration described above as an introduction to CBE. It is important to maintain the problem -based orientation of these courses and it is vital to generate thinking- based material. Within this framework, we will examine two approaches.

The first is a design-based approach in which first year students can be asked to:

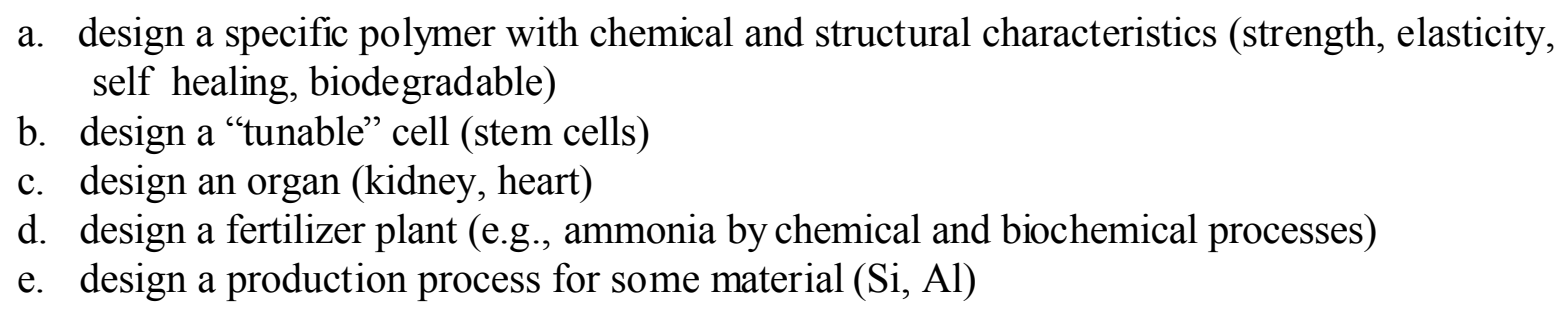

Proceedings of the 2003 American Society for Engineering Education Annual Conference \& Exposition Copyright (C) 2003 American Society for Engineering Education 


\section{Session 2613}

f. design a drug delivery device

In the process, the questions will reveal the material needed to proceed and set up the curriculum. Students will need to develop some background. Only a limited set of questions can be addressed but with proper guidance, they can be answered. The emphasis will be on the molecular basis for the solution of the problem. Note that the questions relate to material with which most students have some familiarity.

The second approach is to develop hands-on first- year design projects, possibly in combination with the "questions" raised above. (Fuel cell, battery, microbrewery, separations, biotransformations). As an example : how to produce alcohol from a synthetic vs. a biological route. The students can explore both options and design a system to accomplish the goal, then compare yields, mass balances, etc. at the preliminary level to get them thinking about the issues, similarities, energy balances and costs, etc.

\section{Second Year}

We propose to modify the second year sequences by replacing the Physical Chemistry with Organic chemistry, and requiring that the advanced chemistry elective in the spring be biochemistry . Physical Chemistry will be delayed until the Junior year. The courses covering Thermodynamics and Process Calculations (CBE 10,11) will be modified in content. The traditional first course is devoted to steady-state material and energy balances, with thermodynamics providing the background for energy balances and transformations (work, heat). It is proposed to have as primary threads a living organism and a chemical plant. Other threads can be introduced as secondary threads at this stage, but in subsequent years more detail can be examined. For these both mass and energy balances can be demonstrated. By recognizing that the biological systems are isothermal and energy sources are chemical or photochemical, an interesting comparison can be made with traditional non isothermal chemical systems. Biological engines can be compared with thermal engines. The second course covers the application of the chemical potential approach to physical and chemical equilibrium. The modifications will include the biochemistry- relevant to organisms (e.g. ATP and consequences). There will be less emphasis on traditional phase equilibrium and more on electrochemical phenomena, membranes, ionic solutions. The enthalpy balances will be extended to include biochemical oxidations, enzyme binding, protein folding/unfolding. The use of $\Delta \mathrm{G}$ in biochemical cycles will be included. The systems to be studied can now be extended to include cells and commodity chemical plants. The plan is to increase gradually the number of threads until all can be considered by senior students. A pilot version of CBE 10 and 11 will be prepared for 2002-2003 and the full version offered in 2003-2004.

\section{Laboratory Development : New Junior Year Laboratory}

A sophomore laboratory was introduced in 1999 to provide students with experience in making simple measurements using mass and volume measurements together with appropriate sensors and evaluating their associated measurement errors. Presentation skills, written and oral, are part of the course. The existing course includes a design component. Students are asked to analyze a system and determine what data are necessary to be able to control the process, in this case an enzymatic reaction. The parameters (temperature, $\mathrm{pH}$, temperature, substrate concentration, etc)

Proceedings of the 2003 American Society for Engineering Education Annual Conference \& Exposition Copyright (C) 2003 American Society for Engineering Education 


\section{Session 2613}

are measured. With this as a basis, we plan to extend this approach using more systems and more sensors. These sensors will include in addition to those mentioned above, pressure, dissolved oxygen, turbidity, optical absorption, and a variety of biosensors. With this background, a new junior level laboratory will be developed to further the integration under the existing paradigm and take advantage of the developing professional competence of the students.

The purpose of this new laboratory is to examine chemical and biological transformations of systems designed to produce the same final product. These examples will be run in parallel and incorporate the sensor experiences from the sophomore lab. The students will be divided into teams to address the parallel paths to reach the product. Underlying concepts to be addressed within the laboratory work will include: mass balances, system design, yields and efficiency, green chemistry considerations, product cost, ease of operation, and related issues. At the end of the semester, the projects would be completed in the form of a comprehensive laboratory report and presented to the other students and faculty for critique. Specific examples of parallel laboratory concepts that would be provided to each team of students would include the following:

Polymerization Reactions - Study and synthesis of the kinetics and characterization of polymers formed via traditional chemical catalysis versus biocatalysis. Contrast the benefits and limitations of both approaches on the basis of yield, mass balances, chemical selectivity, impurities and the related issues outlined above. Specific polymers that can be considered include vinyl polymerizations (carried out by traditional free radical chemistry or via peroxidase-catalyzed biochemical reactions) including a water-soluble product such as polyacrylic acid and a water insoluble product such as polystyrene. Reactions can be set up and run in the laboratory after the system design and assembly is complete. The reaction products can be characterized (e.g., GPC, NMR and MALDI, purity assessed, and kinetics considered during the process).

Energy Harvesting from Solar Energy Collection and Conversion The team of students could design a solar energy collection system and do so by comparing traditional methods (e.g. photovoltaics) and compare this with a biological method (examples could include bacteriorhodopsin or phycobiliproteins). The team would design the complete system, implement the system, and then characterize energy recovery in terms of efficiency, light intensity variables, wavelength of coverage and related issues within the context of the broader objectives outlined earlier.

Alcohol Production - This example would provide the laboratory experience initiated in the first year, but actually be implemented in laboratory experiment form now. This also addresses the threaded concept started in the first year course on comparison between traditional and biological approaches to alcohol production. The students would set up a traditional production scheme for alcohol as well as a fermentation approach, and assess yield and purity along with the other issues outlined earlier.

Other systems might include simple sugar production from agricultural products to compare chemical vs. enzymatic hydrolysis methods to obtain useful sugars.

\section{Summary}

Proceedings of the 2003 American Society for Engineering Education Annual Conference \& Exposition Copyright (C) 2003 American Society for Engineering Education 


\section{Session 2613}

A new approach to the introduction of biological engineering is proposed which treats biological systems under the same paradigm as traditionally employed in Chemical Engineering instruction. This approach also provides an ongoing context for the entire curriculum.

\section{Bibliographic Information}

1. AIChE "75 Years of Progress-A History of AIChE 1908-1983", 1983, pg 12

2. See curricula of a variety of Biological Engineering Departments

3. Chang, Jane, "Redesigning The ChE Curriculum For The Challenges in The $21^{\text {st }}$ century", International Conference on Engineering Education , August 2001, Oslo Norway

4. Khosla ,Chaitan, "Intellectual Border: Two Way Traffic", in "New Voices In Chemistry", C\&E News (ACS)

5. Saltsburg,H Heist ,R H Olsen, $\mathrm{T}$ “Chromatography As A Paradigm For The ChE Laboratory”, ASEE Proceedings ,Annual Conference, 1993, Session 1226

6. Bailey, J Ollis D "Biochemical Engineerin Fundamentals", $2^{\text {nd }}$ ed ,Chapter 7, McGraw-Hill, 1977

7. Rehm, HJ Preve, G in Biotechnology, Vol 1-8 ,1981 ff, H J Rehm \& G Reed eds. Verlag Chemie

\section{Biographical Information}

HOWARD SALTSBURG is Professor of Chemical and Biological Engineering (ChBE) at Tufts. His teaching interests are in laboratory studies and thermodynamics. Research interests include catalysis, fuel cells, and electrochemistry.

MARIA FLYTZANI-STEPHANOPOULOS is Professor of ChBE at Tufts. Her teaching interests include reactor design, transport, and environmental issues.

DAVID KAPLAN is Professor of ChBE., Director of the Biotechnology Center and Chair of the Department of Biomedical Engineering. His teaching interests cover a variety of topics in biology and biotechnology. His research if focused on a variety of biomaterials.

GREGORY BOTSARIS is Professor of ChBE and is acting chair of the department. His teaching interests include thermodynamics and separation processes. His research involves studies of crystal growth and other separation proceses.

KYONGBUM LEE is Assistant Professor of ChBE. His teaching and research are focused on metabolic engineering. 


\section{Session 2613}

Proceedings of the 2003 American Society for Engineering Education Annual Conference \& Exposition Copyright (C) 2003 American Society for Engineering Education

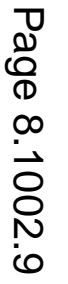

